\title{
Comparison of geometries and electronic structures of polyacetylene, polyborole, polycyclopentadiene, polypyrrole, polyfuran, polysilole, polyphosphole, polythiophene, polyselenophene and polytellurophene
}

\author{
U. Salzner ${ }^{\text {a,1 }}$, J.B. Lagowski ${ }^{\text {b, }}$, P.G. Pickup ${ }^{\text {a, }}$, R.A. Poirier ${ }^{\mathrm{a}, 3}$ \\ 'Department of Chemistry, Memorial University of Newfoundland, St. John's, Nfld., A1B 3X7, Canada \\ ${ }^{b}$ Department of Physics and Physical Oceanography, Memorial University of Newfoundland, St. John's, Nfd., AIB 3X7, Canada
}

Received 19 September 1997; received in revised form 18 May 1998; accepted 22 May 1998

\begin{abstract}
Geometries of monomers through hexamers of cylopentadiene, pyrrole, furan, silole, phosphole, thiophene, selenophene and tellurophene, and monomers through nonamers of borole were optimized employing density functional theory with a slightly modified B3P86 hybrid functional. Bandgaps and bandwidths were obtained by extrapolating the appropriate energy levels of trimers through hexamers (hexamers through nonamers for borole) to infinity. Bandgaps increase with increasing $\pi$-donor strengths of the heteroatom. In general, second period heteroatoms lead to larger bandgaps than their higher period analogs. Polyborole is predicted to have a very small or no energy gap between the occupied and the unoccupied $\pi$-levels. Due to its electron deficient nature polyborole differs significantly from the other polymers. It has a quinoid structure and a large electron affinity. The bandgaps of heterocycles with weak donors $\left(\mathrm{CH}_{2}, \mathrm{SiH}_{2}\right.$ and $\left.\mathrm{PH}\right)$ are close to that of polyacetylene. For polyphosphole this is due to the pyramidal geometry at the phosphorous which prevents interaction of the phosphorus lone pair with the $\pi$-system. The bandgap of polypyrrole is the largest of all polymers studied. This can be attributed to the large $\pi$-donor strength of nitrogen. Polythiophene has the third largest bandgap. The valence bandwidths differ considerably for the various polymers since the avoided crossing between the flat HOMO - 1 band and the wide HOMO band occurs at different positions. The widths of the wide HOMO bands are similar for all systems studied. All of the polymers studied have strongly delocalized $\pi$-systems.
\end{abstract} All rights reserved.

Keywords: Electronic structures; Geometries; Polyborole; Polycyclopentadiene; Polyfuran; Polysilole; Polyphosphole; Polyselenophene; Polytellurophene

\section{Introduction}

Due to their chemical stability, their high conductivity upon doping, and their non-linear optical properties, polypyrrole (PPy) and polythiophene (PTh) are among the most widely studied conjugated organic polymers, experimentally [1-4] and theoretically [5-9]. Since PPy and PTh have relatively large bandgaps, $2.85[10]$ and $2.0 \mathrm{eV}$, [11] respectively, the neutral forms are insulators. Intensive research has

\footnotetext{
* Corresponding author. Tel.; +1 709737 2667; fax: +1 709737 8937; e-mail: jolantal@smaug.physics.mun.ca

${ }^{1}$ Tel.: +1 709737 2151; fax: +1 709737 3702; e-mail: uli@smaug. physics.mun.ca. Present address: Department of Chemistry, Bilkent University, 06533 Bilkent, Ankara, Turkey. Tel.: +903122664000 , ext. 2122; fax: +903122664579; e-mail: salzner@ fen.bilent.edu.tr

${ }^{2}$ Tel: + 17097378657 ; fax: + 17097373702 ; e-mail: ppickup@morgan. ucs.mun.ca

${ }^{3}$ Tel.: + 1709737 8609; fax: + 1709737 3702; e-mail: rpoirier@morgan. ucs.mun.ca
}

been dedicated to chemical modification of these systems with the aim to decrease the bandgaps [4].

Less is known about polycyclopentadiene (PCp) and polyfuran (PFu) and higher period analogs of PTh, polyselenophene (PSe) and polytellurophene (PTe). In the case of $\mathrm{PFu}$ this is caused by the experimental difficulties involved with synthesis and with doping $[12,13]$. However, high quality $\mathrm{PFu}$ has been produced. The bandgap was determined to be $2.35 \mathrm{eV}$ [12] and conductivity upon doping of $10^{2} \mathrm{~S} / \mathrm{cm}$ was reported [14]. This has to be compared to maximal conductivities of $500 \mathrm{~S} / \mathrm{cm}$ for PPy [3] and $2000 \mathrm{~S} / \mathrm{cm}$ for PTh [4]. The bandgap of polyselenophene (PSe) was found to be the same as that of PTh, $2.0 \mathrm{eV}$ [15] but the conductivity was only $3.7 \times 10^{-2}$. Polytellurophene was generated readily at potentials lower than those required for thiophene, but the conductivity after doping was low, $7.6 \times 10^{-6} \mathrm{~S} / \mathrm{cm}[16]$.

This shows that conceptually similar systems can differ vastly in their electrical properties, chemical stability and ease 
of polymerization. It is difficult to decide experimentally whether low conductivities are caused by intrinsic differences in electronic structure or by short conjugation lengths due to limited polymerization, disorder or ring opening.

In this paper we compare properties of polyacetylene (PA), 1, polyborole ( $\mathrm{PBo}$ ), 2, polycyclopentadiene ( $\mathrm{PCP}$ ), 3, polypyrrole (PPy), 4, polyfuran ( $\mathrm{PFu}$ ), 5, polysilole (PSi), 6, polyphosphole $(\mathrm{PPh}), \mathbf{7}$, polythiophene $(\mathrm{PTh}), \mathbf{8}$, polyselenophene (PSe), 9, and polytellurophene (PTe), 10, theoretically. The well-studied systems PA, PPy, PTh are used for comparison and are discussed only briefly. The properties of the less investigated systems are evaluated in comparison with PA, PPy and PTh. This approach allows us to investigate systems with different chemical stabilities in the absence of disorder or limited conjugation lengths.

\section{Methods}

Monomers through hexamers of butadiene, cyclopentadiene, pyrrole, furan, silole, phosphole, thiophene, selenophene and tellurophene, and monomers through nonamers of borole (Fig. 1) were optimized in planar geometry and with trans oriented monomer units employing density functional theory (DFT) with a slightly modified hybrid functional (see below). The phosphole ring is slightly puckered but the carbon backbone was kept planar in analogy with the other systems. All calculations were done with Gaussian 94 [17].

The calculations were carried out using the CEP-31G* keyword in Gaussian 94 [18]. This requests Stephens/ Basch/Krauss effective core potentials (ECPs) and split valence basis sets [19] augmented with polarization functions on heavy atoms. Since the CEP- $31 \mathrm{G}^{*}$ basis is not available for Se and Te, we used the Dunning 95 basis set [20] on second period atoms and Los Alamos ECPs plus double zeta basis sets [21-23] for higher period atoms instead (LANL2DZ keyword in Gaussian 94) [18]. To assess the basis set effect, we optimized the thiophene oligomers with both basis sets. LANL2DZ yields a bandgap slightly smaller $(0.09 \mathrm{eV})$ than CEP-31G*. HOMO and LUMO levels tend to be about $0.5 \mathrm{eV}$ lower than with CEP-31G** Single and double $\mathrm{C}-\mathrm{C}$ bond lengths shorten by up to $0.02 \mathrm{~A}, \mathrm{C}-\mathrm{S}$ bond lengths increase by $0.05 \AA$, bond angles differ by up to $2^{\circ}$.

Polymer properties were obtained by plotting results for trimers through hexamers against inverse chain lengths and extrapolating to infinity. Since the geometry switches from aromatic to quinoid for borole pentamers, we used hexamers through nonamers for extrapolation of borole systems. The energy levels arising from the five $\pi$-orbitals of five-ring heteroaromatics (Fig. 2) are plotted in Figs. 3-6 for pyrrole, thiophene, tellurophene and borole oligomers.

Vertical ionization potentials (IPs) and electron affinities (EAs) were estimated from extrapolated negative HOMO and LUMO energies, respectively. Vertical excitation energies $\left(\lambda_{\max }\right)$ were calculated from extrapolated HOMOLUMO gaps and compared to the corresponding $\lambda_{\max }$ in the

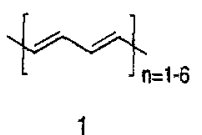<smiles>CC(C)(C)C1=CC=C(C2=CC=C(C(C)(C)C)C2)C1</smiles>

3

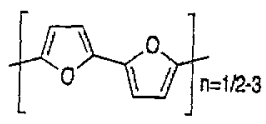

5

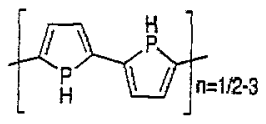

7

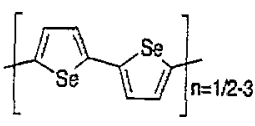<smiles></smiles>

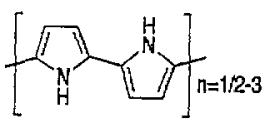

4

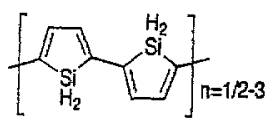

6
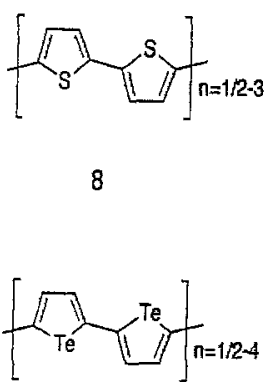

10
Fig. 1. Structures of polyacetylene 1 , polyborole 2 , polycyclopentadiene 3 , polypyrrole 4 , polyfuran 5 , polysilole 6 , polyphosphole 7 , polythiophene 8 , polyselenophene 9 , polytellurophene 10 .
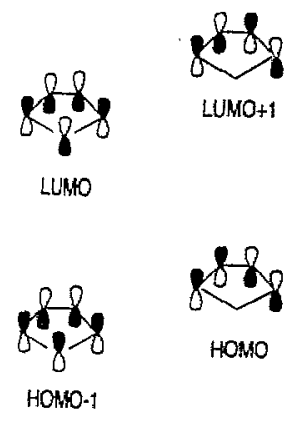

$$
\text { og }
$$

Fig. 2. The $\pi$-orbitals of cyclopentadiene derivatives.

observed absorption spectra of oligomers. Using HOMOLUMO gaps to estimate $\lambda_{\max }$ is analogous to estimating bandgaps from band structure calculations employing Bloch orbitals. Both approaches provide the energy necessary to create free charge carriers, neglecting electronic relaxation effects and do not yield the energy of excitonic excitations [24].

At the LSDA level, bandgaps are underestimated by at least $40 \%[5,25,26]$. This has become known as the bandgap 


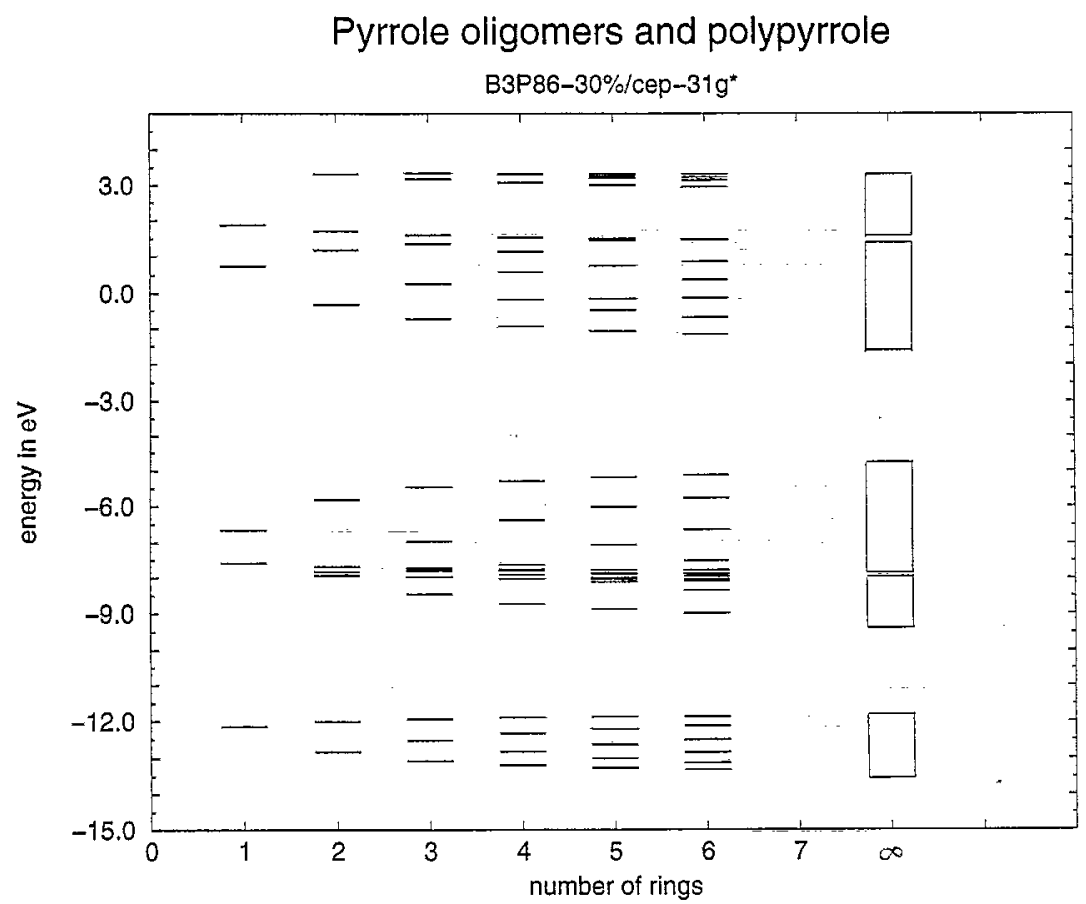

Fig. 3. Development of the band structure of polypyrrole from energy levels of monomer through hexamer of pyrrole.

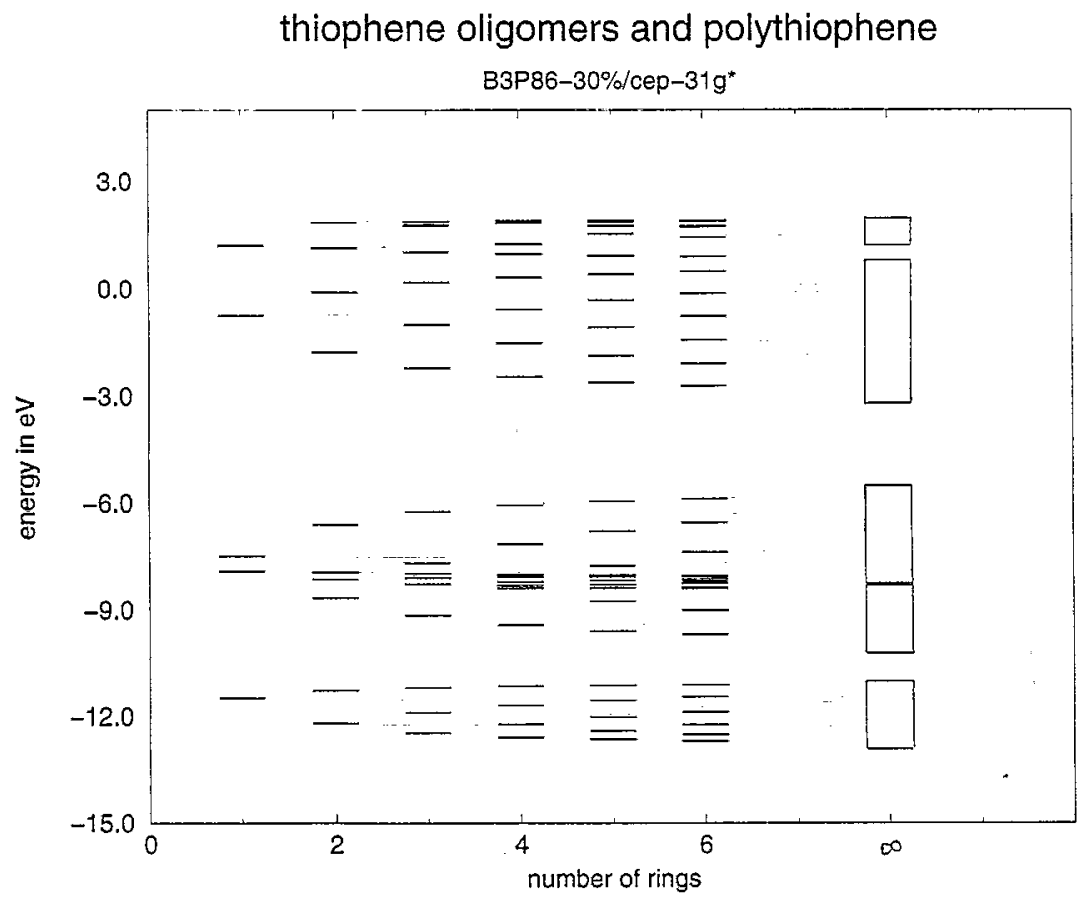

Fig. 4. Development of the band structure of polythiophene from energy levels of monomer through hexamer of thiophene.

problem of density functional theory. The origin of the bandgap problem, i.e. whether it arises from limitations of the LSD approximation or whether it is inherent to DFT, has been investigated in numerous publications [25-29]. The majority of analyses led to the conclusion that the bandgap problem is due to the derivative discontinuity in the exchange correlation functional with respect to particle number and therefore inherent to DFT. In contrast, Williams and von Barth [30] and Baerends and Gritsenko [31] argue that DFT is a powerful tool for approximating absorption spectra and that problems with DFT orbital energies are caused by the poor asymptotic behavior of the density within the LSD approximation and the incomplete cancellation of the selfinteraction energy.

We have examined HOMO and LUMO energy levels, HOMO-LUMO gaps and extrapolated bandgaps employing hybrid functionals empirically $[32,33]$. Inclusion of HartreeFock $(\mathrm{HF})$ exchange in the exchange correlation functional 


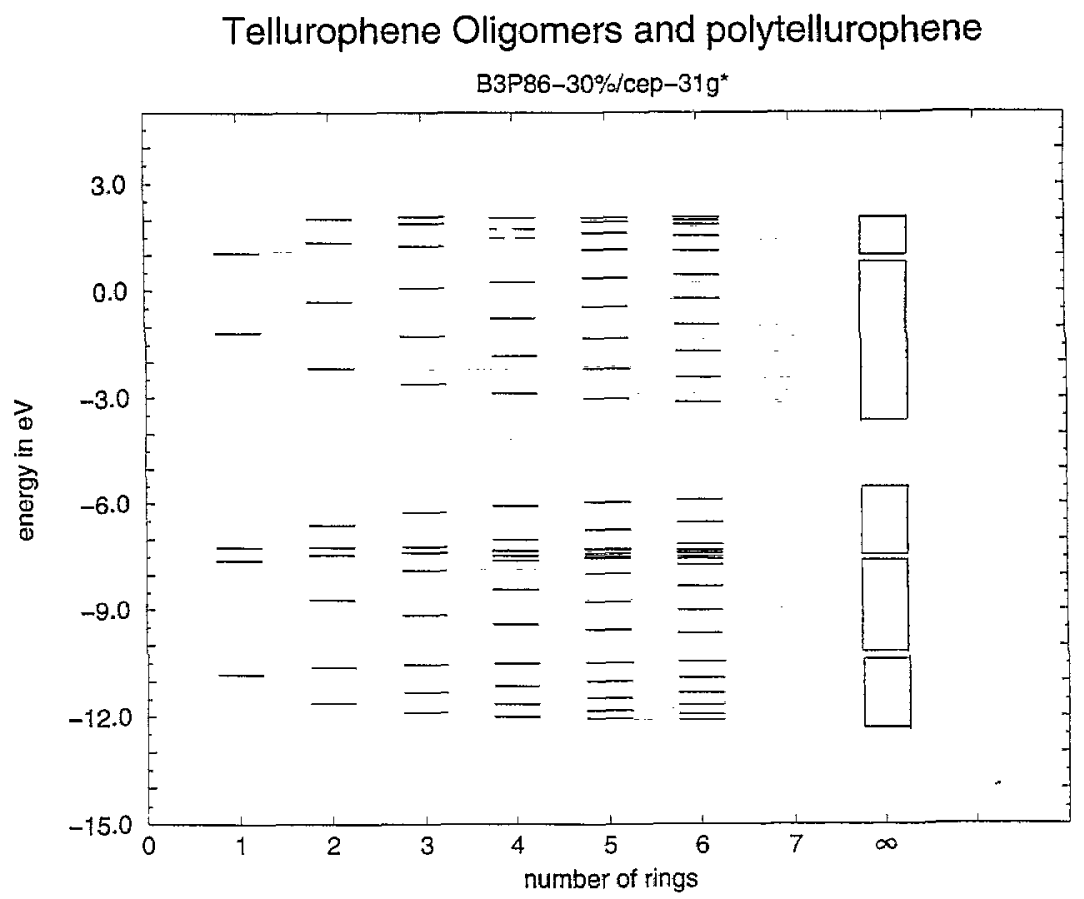

Fig. 5. Development of the band structure of polytellurophene from energy levels of monomer through hexamer of tellurophene.

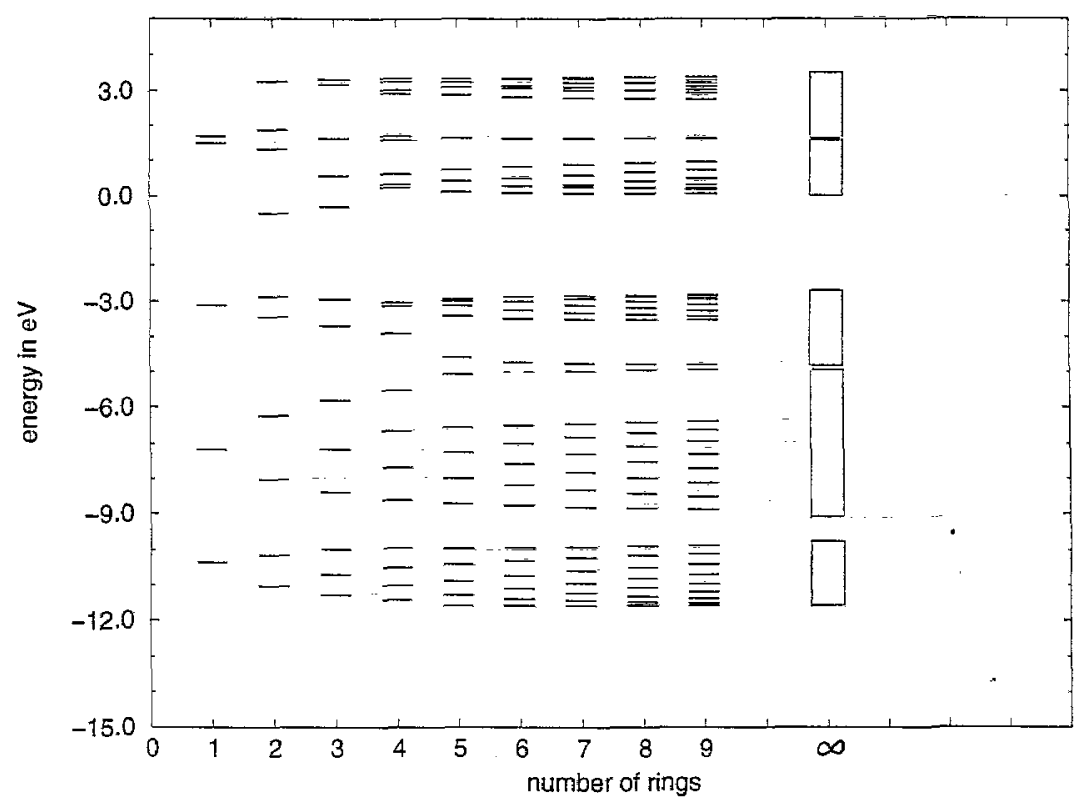

Fig. 6. Development of the band structure of polyborole from energy levels of monomer through nonamer of borole.

leads to a considerable improvement of energy gaps over those obtained with LSDA. By trial and error we established that a weight of $30 \%$ for the HF exchange leads to better agreement of HOMO-LUMO gaps with experimental excitation energies than with the B3LYP [18] or B3P86 [18] functionals which include $20 \% \mathrm{HF}$ exchange. With the B3P86-30\% functional extrapolated bandgaps of polythiophene, polypyrrole, polyfuran and polythiazole agree to within $\pm 0.1 \mathrm{eV}$ with experimental bandgaps [32]. The success of the approach is partially due to error cancellation since HOMO and LUMO energies underestimate IPS and EAs by about $1 \mathrm{eV}$ [32]. All calculations in the present paper were carried out employing the B3P86-30\% functional. Since the polymers under investigation have very similar electronic structures, we expect the predicted bandgaps to be accurate to at least within $\pm 0.2 \mathrm{eV}$.

Bandwidths were obtained as orbital energy differences between the appropriate extrapolated orbital energies. Each of the five $\pi$-orbitals (compare Fig. 2) of the monomers gives rise to a band in the polymers. Figs. 3-6 show how the bands develop gradually for pyrrole, thiophene, tellurophene and borole. Extrapolated bands for polymers are also depicted. 


\section{Results}

\subsection{Bandgaps}

Table 1 summarizes bandgaps, ionization potentials (IPs), electron affinities (EAs), and bandwidths for PA, PBo, PCp, PPy, PFu, PSi, PPh, PTh, PSe and PTe. Experimental data are included where available. The extrapolated bandgaps for the polymers with exception of polyborole range from 1.39 to $3.16 \mathrm{eV}$. Polyborole has a vanishingly small bandgap of about $0.12 \mathrm{eV}$ or less. The extrapolated LUMO level lies actually below the extrapolated HOMO level for polyborole. A small bandgap may therefore arise due to an avoided crossing. The widely used polymer PPy has the largest bandgap, $3.16 \mathrm{eV}$, of all systems studied. PTh has the third largest bandgap, $2.30 \mathrm{eV}$.

Theoretical bandgaps for PA, PPy, PFu and PTh are between 0.11 and 0.31 higher than experimental gaps. We argued in a recent publication [32] that theoretical bandgaps calculated for isolated chains are expected to be about $0.2 \mathrm{eV}$ larger than condensed phase values. After correcting for this effect, our theoretical bandgaps agree to within $0.11 \mathrm{eV}$ with experiment. This success in predicting bandgaps involving a range of different heteroatoms gives us confidence that predictions for unknown systems are reliable.

\subsection{Bandwidths}

Table 1 lists HOMO, valence and conduction bandwidths as obtained from extrapolation of the appropriate oligomer energy levels (compare Figs. 3-6). The lowest $\pi$-band which originates from the all bonding $\pi$-orbital is much lower in energy and well separated from the next two $\pi$-bands for PPy, PTh and PBo. For PTe there is almost no gap between these bands.

The HOMO orbitals give rise to wide bands (which run down in band structure diagrams), the HOMO - 1 orbitals generate narrow bands (which run up slightly). Due to the large splitting of the energy levels arising from the HOMOs as the chains get longer, the HOMO - 1 orbital energies already lie within the levels arising from the HOMOs for trimers of PPy, PTh and PTe. This leads to avoided crossings in band structure diagrams. Since bandwidths of polymers are determined using the uppermost band, valence bandwidths from band structure calculations include levels arising from the HOMO -1 at the beginning of the Brillouin zone $(k=0)$ and levels arising from the HOMO at the end of the Brillouin zone $(k=\pi / a)$. The HOMO -1 bands are very narrow and contribute little to bandwidths (for relative magnitudes of the HOMO and valence bands see Table 1). Therefore, the position of the avoided crossing has a major influence on the valence bandwidths (compare Figs. 4 and 5 for $\mathrm{PTh}$ and $\mathrm{PTe}$ ). For $\mathrm{PBo}$ no avoided crossing in the valence region occurs since the band corresponding to the HOMO -1 of the other polymers is empty and forms the valence band (compare Fig. 7). HOMO and valence bandwidths are therefore identical for PBo.

Since we intend to use bandwidths as a measure for delocalization [34], we will use HOMO bandwidths rather than valence bandwidths to compare delocalization in different polymers. The HOMO bands are wider than the valence bands and differ by only $0.77 \mathrm{eV}$ for the different polymers. PCp has the largest HOMO bandwidth $(5.06 \mathrm{eV})$ and PBo has the smallest $(4.13 \mathrm{eV})$. HOMO bandwidths of the remaining polymers lie within $0.23 \mathrm{eV}$. These similar values indicate very similar degrees of delocalization of all systems studied here.

The LUMO and LUMO + 1 give rise to two unoccupied bands. For PPy a gap opens above the LUMO band but for PTh and PTe no such splitting of the levels is visible in Figs. 4 and 5. To estimate valence bandwidths we have nonetheless included only the energy levels having their origin in the LUMO of the monomer.

The largest conduction bandwidths were obtained for PCp, PSe and PTe (around $4 \mathrm{eV}$ ), even after correcting for a basis set effect of about $0.3 \mathrm{eV}$ for the latter two. PBo and PPy have the smallest conduction bandwidths. The conduction band of PA is wider than those of the heterocyclic polymers. The difference is that all $\pi$-levels form one band in PA; in contrast, the $\pi$-levels give rise to two separate bands for the heterocyclic polymers. For $\mathrm{PCp}, \mathrm{PTh}, \mathrm{PSe}$ and $\mathrm{PTe}$, there is

Table 1

Theoretical and experimental bandgaps $\left(E_{\mathrm{g}}\right)$, IPs EAs, and theoretical bandwidths $\left(B_{\mathrm{w}}\right)$ for PA, PBo, PCp, PPy, PFu, PSi, PPh, PTh, PSe and PTe

\begin{tabular}{|c|c|c|c|c|c|c|c|c|}
\hline & $E_{\mathrm{g}}$ theor. & $E_{\mathrm{g}}$ exp. & IP theor. & IP exp. & EA & $B_{\mathrm{w}}$ HOMO & $B_{\mathrm{w}}$ val. & $B_{\mathrm{w}}$ con. \\
\hline $\mathrm{PA}$ & 1.57 & $1.48[45]$ & 5.02 & $4.5 \pm 0.1[71]$ & 3.45 & 5.96 & 5.96 & 5.22 \\
\hline PBo & 0.12 & & 4.95 & & 4.83 & 4.13 & 4.13 & 2.13 \\
\hline $\mathrm{PCp}$ & 1.58 & & 4.61 & & 2.97 & 5.06 & 4.45 & 4.09 \\
\hline PPy & 3.16 & $2.85[10]$ & 4.77 & $4.0[11]$ & 1.61 & 4.62 & 3.08 & 2.98 \\
\hline PFu & 2.67 & $2.35[12]$ & 5.31 & & 2.63 & 4.74 & 3.77 & 3.48 \\
\hline PSi & 1.39 & & 5.14 & & 3.75 & 4.85 & 4.32 & 3.16 \\
\hline $\mathrm{PPh}$ & 1.49 & & 5.23 & & 3.73 & & & \\
\hline PTh (CEP) & 2.30 & $2.0[46]$ & 5.50 & $5.0[11]$ & 3.20 & 4.68 & 2.75 & 4.00 \\
\hline PTh (LAN) & 2.21 & & 5.76 & & 3.55 & 4.81 & 2.74 & 4.31 \\
\hline $\mathrm{PSe}$ & 2.06 & & 5.64 & & 3.58 & 4.78 & 2.41 & 4.44 \\
\hline PTe & 1.87 & & 5.52 & & 3.63 & 4.64 & 1.90 & 4.43 \\
\hline
\end{tabular}




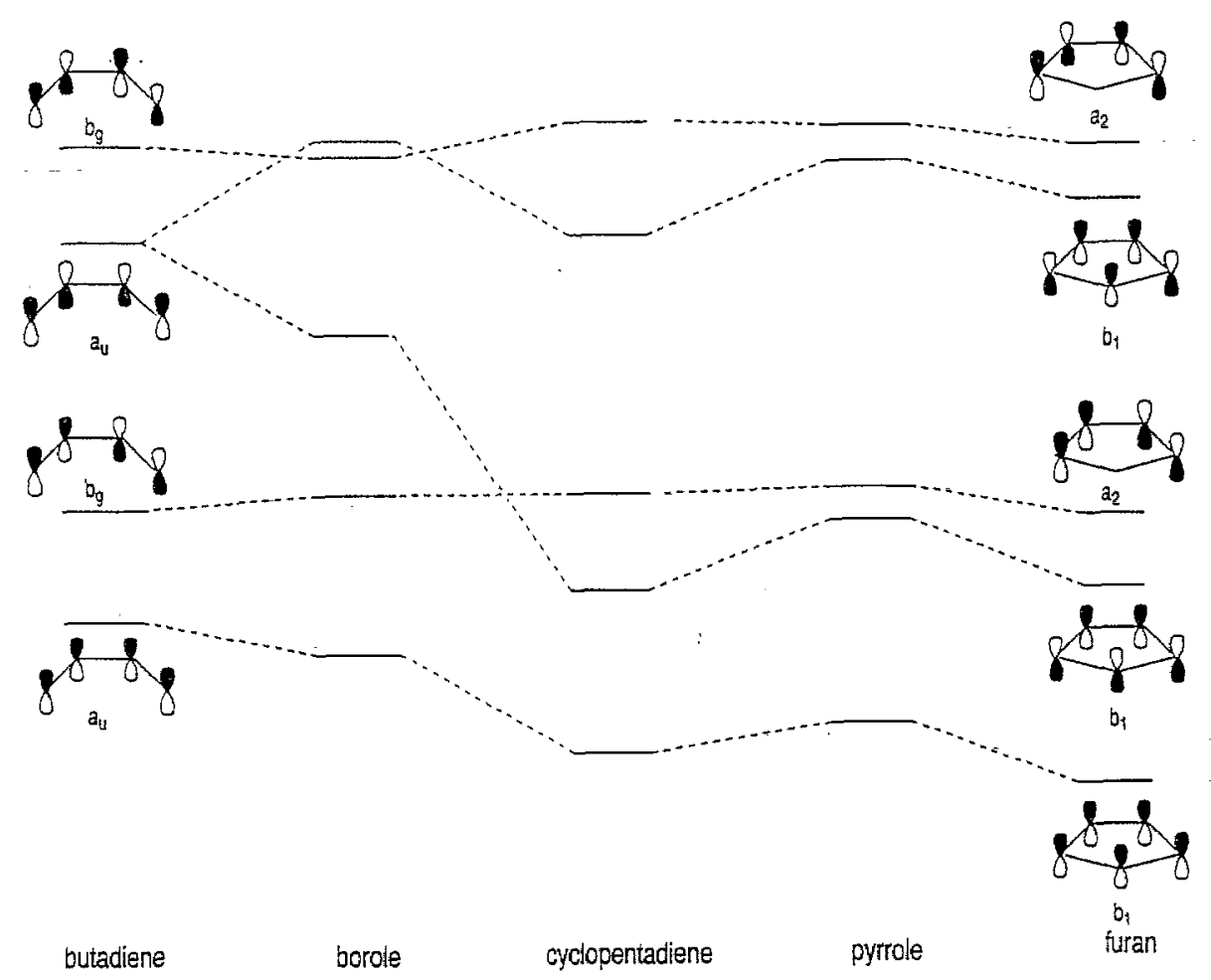

Fig. 7. Comparison between energy levels of second period heterocycles.

almost no gap between the conduction band and the next higher $\pi$-band. For PPy, PFu and PSi, there is a large gap between the conduction band and the next higher $\pi$-band.

\subsection{Ionization potentials (IPs) and electron affinities (EAs)}

Most of the polymers studied have IPs between 5.1 and $5.5 \mathrm{eV}$. Note that there is a basis set effect of $0.26 \mathrm{eV}$ for PTh. Assuming it would be similar for PSe and PTe, the IPs of these polymers were 5.38 and $5.26 \mathrm{eV}$, respectively. The IPs for PBo, PCp and PPy are lower, 4.95, 4.61 and $4.77 \mathrm{eV}$.

The EAs vary over a much wider range than the IPs. PPy has the smallest EA, followed by PFu and PCp. EAs of PTh, PSe and PTe are very similar and larger than those of the former compounds. PSi and PPh have EAs 0.53 and $0.55 \mathrm{eV}$ larger than PTh, which suggests that they are possible candidates for stable n-doped polymers. Finally, PBo has a large $\mathrm{EA}, 4.83 \mathrm{eV}$, due to its electron deficient nature.

\subsection{Geometries}

In Table 2 bond lengths are summarized for the inner rings of hexamers of our heterocycles and for the central unit of $\mathrm{C}_{24} \mathrm{H}_{26}$. Where available, comparison is made to other work. The only system for which X-ray data exist for hexamers is thiophene. With the CEP- $31 \mathrm{G}^{*}$ basis set, the bonds in sexithiophene are up to $0.026 \AA$ longer than the experimental values [35]. The bond angles (not shown) agree very closely (to within $0.7^{\circ}$ ) with experiment. With exception of the $\mathrm{C}-$ $S$ bond, LSDA [36] and DFT/hybrid geometries are almost identical. Compared to HF theory [9], DFT yields longer $\mathrm{C}=\mathrm{C}$ double bonds and shorter interring bonds. Thus, at $\mathrm{HF}$, $\pi$-electrons are more localized. This is most likely due to the neglect of electron correlation. The unpolarized LANL2DZ basis set leads to shorter interring $\mathrm{C}-\mathrm{C}$ and intraring $\mathrm{C}-\mathrm{C}$ and $\mathrm{C}=\mathrm{C}$ bonds but longer $\mathrm{C}-\mathrm{S}$ bonds. The intraring $\mathrm{C}-\mathrm{C}$ and $\mathrm{C}=\mathrm{C}$ bonds are in closer agreement, the $\mathrm{C}-\mathrm{S}$ and $\mathrm{C}-\mathrm{C}$ bonds are in poorer agreement with experiment than with the CEP$31 \mathrm{G}^{*}$ basis set. The bond length alternation is underestimated with DFT, especially with the LANL2DZ basis set, and overestimated with $\mathrm{HF}$ theory compared to experiment.

For PPy and PFu, geometries can be compared to HF and LSDA data only. For the aromatic pyrrole and furan rings, HF theory seems to underestimate electron delocalization considerably. This results in shorter $\mathrm{C}=\mathrm{C}$ and longer $\mathrm{C}-\mathrm{C}$ bonds. LSDA and DFT/hybrid results compare favorably.

The bond length alternation $\Delta$ is given as the difference between the double bond and the longer single bond (intraring or interring). For PA we obtain single bond lengths of $1.444 \AA$ and double bond lengths of $1.380 \AA$. The bond length alternation is $0.064 \AA$. With the exception of sexiborole, all hexamers are aromatic. This means that the interring bond is a single bond. For PPy, PFu, PTh, PSe and PTe, the intraring single bonds are shorter that the interring bonds. For PPh both single bonds are about the same length. In sexicyclopentadiene and sexisilole, the bonds connecting the rings are shorter than the intraring single bonds. 
Table 2

Bond lengths of central units of $\mathrm{C}_{24} \mathrm{H}_{26}$ and hexamers of heterocycles

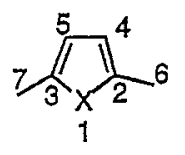

\begin{tabular}{|c|c|c|c|c|c|}
\hline & $C_{2}-X$ & $\mathrm{C}_{2}=\mathrm{C}_{4}$ & $\mathrm{C}_{4}-\mathrm{C}_{5}$ & $\mathrm{C}_{2}-\mathrm{C}_{6}$ & $\Delta$ \\
\hline $\mathrm{PA}$ & & 1.380 & 1.444 & & 0.064 \\
\hline PBo & 1.573 & 1.471 & 1.382 & 1.388 & 0.089 \\
\hline PCp & 1.523 & 1.386 & 1.454 & 1.447 & 0.068 \\
\hline PPy & 1.380 & 1.402 & 1.429 & 1.457 & 0.055 \\
\hline $\mathrm{HF}$ [9] & 1.371 & 1.371 & 1.452 & 1.449 & 0.081 \\
\hline LSDA [36] & 1.406 & 1.405 & 1.433 & 1.462 & 0.057 \\
\hline $\mathrm{PFu}$ & 1.366 & 1.387 & 1.435 & 1.449 & 0.062 \\
\hline $\mathrm{HF}[9]$ & 1.375 & 1.351 & 1.439 & 1.432 & 0.088 \\
\hline $\mathrm{PSi}$ & 1.894 & 1.389 & 1.463 & 1.448 & 0.074 \\
\hline $\mathrm{PPh}$ & 1.846 & 1.389 & 1.446 & 1.451 & 0.062 \\
\hline PTh (CEP) & 1.752 & 1.396 & 1.427 & 1.459 & 0.063 \\
\hline (LAN) & 1.804 & 1.381 & 1.422 & 1.436 & 0.055 \\
\hline HF [9] & 1.745 & 1.358 & 1.427 & 1.454 & 0.096 \\
\hline LSDA [36] & 1.729 & 1.395 & 1.428 & 1.461 & 0.066 \\
\hline Exp. [35] & 1.73 & 1.37 & 1.41 & 1.45 & 0.08 \\
\hline PSe & 1.921 & 1.382 & 1.422 & 1.436 & 0.054 \\
\hline HF [9] & 1.884 & 1.379 & 1.449 & 1.476 & 0.097 \\
\hline $\mathrm{PTe}$ & 2.100 & 1.384 & 1.423 & 1.437 & 0.053 \\
\hline
\end{tabular}

\section{Discussion}

\subsection{Polyacetylene}

Polyacetylene has been investigated intensively $[6,37-45]$ (this list of references is far from complete). Upon doping, stretched aligned PA reaches conductivities of $10^{6} \mathrm{~S} / \mathrm{cm}$ [46], close to that of copper. On a conductivity to weight ratio, the conductivity of PA exceeds that of copper. The bandgap determined as the onset of photoconductivity is 1.48 $\mathrm{eV}$ [45]. This is slightly higher than the absorption onset and lower than $\lambda_{\max }$ of $1.9 \mathrm{eV}$ [39].

We obtained a bandgap of $1.57 \mathrm{eV}$ for PA. After correction for the difference between gas phase and condensed phase of $0.2 \mathrm{eV}$ this is only $0.09 \mathrm{eV}$ lower than the experimental value. Valence and conduction bandwidths are 5.96 and $5.22 \mathrm{eV}$, respectively. The IP is $0.48 \mathrm{eV}$ lower and the EA is $0.25 \mathrm{eV}$ higher than that of PTh.

\subsection{Polyborole}

Polyborole is not known experimentally. The pentaphenylborole monomer, however, has been synthesized [47]. It is deep blue with an absorption maximum of $567 \mathrm{~nm}$ or 2.19 $\mathrm{eV}$. This agrees reasonably well with our calculated value of $2.98 \mathrm{eV}$ for the unsubstituted monomer in the gas phase. Borole is antiaromatic and has the same color as the isoelectronic, antiaromatic pentaphenylcyclopentadienyl cation. In air, pentaphenylborole is instantly oxidized. Since n-doping would make borole isoelectronic with the other heterocyclic systems in this study, the $\mathrm{n}$-doped form might be more stable than the neutral form. This is supported by the recent experimental discovery of a planar five-membered ring structure of the dianion of 1,2-diborata-4-boracyclopentadiene [48] which is isoelectronic with borole. Since boron carried the disilylamino substituent, the system can be considered to be partially $\mathrm{n}$-doped. Thus, $\mathrm{n}$-doped polyborole might be stable enough to be obtained experimentally.

In Fig. 7, orbital levels of heterocycles of second period elements are compared to those of butadiene. It can be seen that the empty $\pi$-orbital of boron leads to a splitting of the butadiene LUMO in two levels. The splitting of the LUMO level is, however, smaller than for the other heterocycles, where the $b_{1}$ level drops below the $a_{2}$ level and becomes the HOMO - 1 . Thus, compared to butadiene the result is a lowering of the LUMO. This leads to the long wavelength absorption observed for borole.

Table 3 summarizes bond lengths in borole oligomers. Borole through quaterborole have aromatic structures ( single bonds connecting the rings and double bonds between $\mathrm{C} 2$ and $\mathrm{C} 3$, and between $\mathrm{C} 4$ and $\mathrm{C} 5$ ). With five rings the structure changes to quinoid with double bonds connecting the rings and single bonds between $\mathrm{C} 2$ and $\mathrm{C} 3$, and between $\mathrm{C} 4$ and C5. Associated with this structural change is a decrease in bandgap and an increase in valence and conduction bandwidths (compare Fig. 6). For oligomers with more than five rings, the bandgaps and bandwidths develop linearly with $1 /$ $n$. This shows that end effects diminish quickly with increasing chain lengths even for systems with quinoid structures. With sufficient chain length, structural and electronic properties can be calculated with the use of oligomers and extrapolation methods. 
Table 3

Evolution of geometric parameters for borole oligomers with increasing chain length<smiles>C=C1C=CC(=O)C1C</smiles>

\begin{tabular}{|c|c|c|c|c|c|}
\hline & $C_{2}-X$ & $\mathrm{C}_{2}=\mathrm{C}_{4}$ & $\mathrm{C}_{4}-\mathrm{C}_{5}$ & $\mathrm{C}_{2}-\mathrm{C}_{6}$ & $\Delta$ \\
\hline Monomer & 1.590 & 1.363 & 1.525 & & 0.162 \\
\hline Dimer & $1.610 / 1.569$ & $1.378 / 1.373$ & 1.500 & 1.448 & 0.122 \\
\hline Trimer & 1.593 & 1.399 & 1.479 & 1.445 & 0.080 \\
\hline Tetramer & $1.585 / 1.591$ & $1.397 / 1.397$ & 1.465 & 1.434 & 0.068 \\
\hline Pentamer & 1.575 & 1.458 & 1.393 & 1.394 & 0.065 \\
\hline Hexamer & $1.572 / 1.573$ & $1.471 / 1.470$ & 1.382 & 1.388 & 0.089 \\
\hline Heptamer & 1.572 & 1.473 & 1.380 & 1.387 & 0.093 \\
\hline Octamer & $1.572 / 1.572$ & $1.474 / 1.474$ & 1.380 & 1.387 & 0.094 \\
\hline Nonamer & 1.572 & 1.474 & 1.379 & 1.387 & 0.095 \\
\hline
\end{tabular}

HOMO-LUMO gaps of all borole oligomers are very small. Extrapolation of hexamer through nonamer values indicates that occupied and unoccupied $\pi$-levels cross, the HOMO drops $0.12 \mathrm{eV}$ below the LUMO. A small bandgap might thus arise from an avoided crossing or polyborole might be a synthetic metal. Due to its very low lying unoccupied $\pi$-levels it is also a good candidate for $\mathrm{n}$-doping. As outlined above, the stability of the neutral form might be poor, but the n-doped form is expected to be more stable. Polyborole, neutral or $\mathrm{n}$-doped, is predicted to have very interesting electronic properties.

\subsection{Polycyclopentadiene}

Polycyclopentadiene is one of the experimentally less investigated systems. It has been obtained from chemical polymerization of cyclopentadiene and bicyclopentadiene. In dichloromethane, black conducting plastics have been precipitated [49]. Theoretically, it was examined with semiempirical methods [50-52]. Hong et al. found PCp to be quinoid and to exhibit a bandgap of $1.21 \mathrm{eV}$. The small bandgap was attributed to the decreased interference of the $\mathrm{CH}_{2}$ group with the $\pi$-systems compared to $\pi$-donating groups.

The DFT/hybrid oligomer approach predicts the structure of sexicyclopentadiene to be aromatic. Table 4 lists bond length parameters for cyclopentadiene through sexicyclopentadiene. As for terthiophene, the geometry is essentially converged for tercyclopentadiene. The central interring bond is $0.011 \AA$ shorter in the hexamer than in the dimer. This is roughly the same difference as between the thiophene dimer and hexamer $(0.009 \AA)$. The intraring single bond is longer in sexicyclopentadiene than in sexithiophene and the $\mathrm{C}=\mathrm{C}$ bond is shorter. The opposite trend would be expected if $\mathrm{PCp}$ were quinoid. The oligomer approach, however, favors aromatic structures due to end effects. Nonetheless, the case of polyborole (compare previous section) shows that a chain length of five monomer units is long enough to allow for switching from aromatic to quinoid structure. We would expect to see at least slight structural changes toward the quinoid form with increasing chain lengths if the polymer preferred a quinoid structure. This can be observed in Table 3 already between monomer and dimer of borole.

Semi-empirical methods which found PCp to be quinoid seem to favor quinoid structures. For PTh the existence of a quinoid structure (although higher in energy than the aromatic form) is predicted at semi-empirical levels [43]. This was shown to be an artifact with DFT methods [5]. In summary, we do not see any indication that PCp is quinoid but, due to the bias toward aromatic structures with the oligomer approach, a final decision cannot be reached at this point.

The extrapolated bandgap obtained for aromatic $\mathrm{PCp}$ is $1.58 \mathrm{eV}$. This is $0.72 \mathrm{eV}$ less than that of PTh and $1.60 \mathrm{eV}$ less than that of PPy at the same level of theory. The value is, however, still too large to allow for significant intrinsic conductivity. The IP is about $0.89 \mathrm{eV}$ lower than that of PTh and $0.16 \mathrm{eV}$ lower than that of PPy. The EA is $0.23 \mathrm{eV}$ lower than that of PTh and $1.5 \mathrm{eV}$ larger than that of PPy. Therefore, PCp should be p-dopable. The $\mathrm{n}$-doped form is not expected

Table 4

Evolution of geometric parameters for cyclopentadiene oligomers with increasing chain length

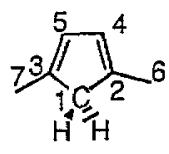

\begin{tabular}{llllll}
\hline & $\mathrm{C}_{2}-\mathrm{C}_{1}$ & $\mathrm{C}_{2}=\mathrm{C}_{4}$ & $\mathrm{C}_{4}-\mathrm{C}_{3}$ & $\mathrm{C}_{2}-\mathrm{C}_{6}$ & $\Delta$ \\
\hline Monomer & 1.513 & 1.368 & 1.480 & & 0.112 \\
Dimer & $1.522 / 1.513$ & $1.380 / 1.368$ & 1.471 & 1.458 & 0.091 \\
Trimer & 1.522 & 1.383 & 1.463 & 1.456 & 0.080 \\
Tetramer & $1.522 / 1.522$ & $1.385 / 1.383$ & 1.457 & 1.449 & 0.072 \\
Pentamer & 1.523 & 1.385 & 1.455 & 1.448 & 0.070 \\
Hexamer & $1.523 / 1.523$ & $1.386 / 1.386$ & 1.454 & 1.447 & 0.088 \\
\hline
\end{tabular}


to be stable. HOMO and conduction bands of PCp are wider than those of PTh and PPy. Therefore, p-doped PCp could, in principle, be a better conductor than PTh and PPy.

\subsection{Polypyrrole}

Polypyrrole has been studied extensively experimentally $[1,2,53,54]$ and theoretically $[6-9,55]$. The bandgap is usually given to be $3.2 \mathrm{eV}$ [11]. A recent investigation by Zotti et al. [10] showed that polymerization of pentamers and heptamers leads to a smaller bandgap, $2.85 \mathrm{eV}$, probably due to a more ordered structure. The highest conductivity achieved for PPy is, to our knowledge, $500 \mathrm{~S} / \mathrm{cm}$ [3]. PPy is stable in the p-doped form but very air sensitive in the neutral form. PPy cannot be n-doped.

We obtain a bandgap of $3.16 \mathrm{eV}$. After correction for the condensed phase effect of about $0.2 \mathrm{eV}$, the predicted bandgap of $2.96 \mathrm{eV}$ is in excellent agreement with the experimental value of 2.85. PPy has the lowest IP and the lowest EA of all polymers studied here. This agrees nicely with experimental observation that PPy can be p- but not n-doped. Although PPy has the smallest HOMO bandwidth of all systems studied here, the conductivity in the p-doped state is high. Thus, the conductivity of none of the other systems in this investigation is expected to be limited by bandwidth.

\subsection{Polyfuran}

Experimental investigation of polyfuran was complicated by the drastic conditions necessary to polymerize furan [12]. As a result $\mathrm{PFu}$ was usually obtained with short conjugation lengths due to ring opening. However, well-ordered material has finally been obtained [56]. Neutral PFu is air stable at room temperature. The bandgap was determined to be 2.35 $\mathrm{eV}$ [12], $0.35 \mathrm{eV}$ larger than that of PTh. This is in excellent agreement with our prediction of $2.67 \mathrm{eV}$, which is 0.37 larger than that for PTh. Conductivities as high as $100 \mathrm{~S} / \mathrm{cm}$ were reported [57]. This is in the range of earlier results for PPy and PTh. Glenis et al., [12], however, found that doped PFu is relatively unstable. Especially in the p-doped state, nucleophilic agents cause ring opening.

The HOMO bandwidth of PFu is slightly larger than those of PTh and of PPy. The conduction band is wider than that of PPy but narrower than that of PTh. Although the IP of furan is higher than that of thiophene, the IP of PFu is predicted to be $0.19 \mathrm{eV}$ lower than that of PTh. This is in qualitative agreement with ab initio calculations [55] which predict the IP of PFu to be $0.06 \mathrm{eV}$ lower than that of PTh. The EA of PFu is $0.57 \mathrm{eV}$ lower than that of PTh and 1.02 $\mathrm{eV}$ larger than that of PPy. We expect therefore that PFu will not be very stable in the n-doped form.

These results show that the electronic properties of ordered infinite chains of PFu are not inferior to those of PTh and PPy. Therefore, higher conductivities can be expected if the quality of the polymer can be further improved. Differences in measured conductivities are probably due to ring opening and limited conjugation lengths.

\subsection{Polysilole}

In 1989 synthesis of polysilole was reported [58] but it was shown later by the same group that the polymer was polydiethenylsilane with four-membered rather than fivemembered rings $[59,60]$. The bandgap was measured to be $2 \mathrm{eV}$. Ab initio calculations are in agreement with experimental findings [61]. At the semi-empirical level, it could be shown that a quinoid structure of polysilole would also have a bandgap of about $2 \mathrm{eV}$ while the aromatic form has a lower bandgap, $1.44 \mathrm{eV}$ [62]. The quinoid form was predicted to about $2 \mathrm{kcal} / \mathrm{mol}$-ring more stable.

As with sexicyclopentadiene we obtain an aromatic geometry for sexisilole. Geometric parameters for monomer through hexamer of silole are summarized in Table 5. Again there is no indication in the structural trends of the oligomers that PSi will switch to a quinoid form with longer chain length. The interring single bond length and the intraring $\mathrm{C}=\mathrm{C}$ bond lengths in sexisilole have the same lengths as those in sexifuran. However, oligomer calculation employed here cannot determine ultimately whether PSi is aromatic or quinoid. The bandgap is $1.39 \mathrm{eV}$, in good agreement with the Hückel value for the aromatic form [62]. The IP of PSi is predicted to be lower than that of PTh and the EA is predicted to be higher. Thus, PSi could be $\mathrm{p}$ - and $\mathrm{n}$-dopable. The HOMO band is $0.17 \mathrm{eV}$ wider than that of PTh, the conduction bandwidth is smaller than that of PTh but wider than that of PPy. Thus, PSi is a good candidate for a conducting polymer.

\subsection{Polyphosphole}

Polyphosphole has not been studied experimentally or theoretically. Oligomers up to quaterphosphole were synthesized and crystal structures were obtained [63]. The structure is twisted with a P-C-C-P dihedral angle around the central

Table 5

Evolution of geometric parameters for silole oligomers with increasing chain length

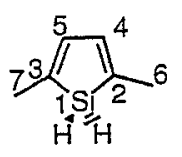

\begin{tabular}{llllll}
\hline & $\mathrm{C}_{2}-\mathrm{Si}$ & $\mathrm{C}_{2}=\mathrm{C}_{4}$ & $\mathrm{C}_{4}-\mathrm{C}_{5}$ & $\mathrm{C}_{2}-\mathrm{C}_{6}$ & $\Delta$ \\
\hline Monomer & 1.880 & 1.368 & 1.497 & & 0.129 \\
Dimer & $1.899 / 1.875$ & $1.379 / 1.371$ & 1.485 & 1.461 & 0.082 \\
Trimer & 1.893 & 1.384 & 1.470 & 1.456 & 0.082 \\
Tetramer & $1.894 / 1.895$ & $1.386 / 1.385$ & 1.467 & 1.451 & 0.082 \\
Pentamer & 1.894 & 1.388 & 1.464 & 1.450 & 0.076 \\
Hexamer & $1.894 / 1.894$ & $1.389 / 1.388$ & 1.463 & 1.448 & 0.088 \\
\hline
\end{tabular}


bond of $25^{\circ}$. The authors concluded that there is no conjugation between the phosphole rings because the interring bonds ( 1.43 and $1.451 \AA$ ) are not shortened. This is a surprising conclusion considering that a normal $\mathrm{C}-\mathrm{C}$ bond is about $1.5 \AA$ long. Also, the central interring bond is $0.2 \AA$ shorter than the outer interring bond. The twist angle also is larger for the olter rings, $49^{\circ}$, than around the central bond, $25^{\circ}$.

In Table 6 theoretical and experimental bond lengths are compared. Although the crystal structure was obtained for quaterphosphole end capped by $\mathrm{Br}$ and substituted with phenyl rings at $P$ and methyl groups at $C_{3}$ and $C_{4}$, the agreement is good. The intraring $\mathrm{C}=\mathrm{C}$ and $\mathrm{C}-\mathrm{C}$ bonds differ only by $0.002-0.004 \AA$. The theoretical $\mathrm{C}-\mathrm{P}$ bond and the central interring bond are $0.02 \AA$ longer. The inner rings in calculated planar quaterphosphole are symmetric, while they are unsymmetrical in the experimental structure. This is probably due to the twisting of the substituted quaterphosphole.

Our calculated bandgap for polyphosphole is $1.49 \mathrm{eV}$. This is the third lowest bandgap of all systems in this study. This can be explained by effective conjugation along the chain in combination with the inter pair effect. Phosphorous, like other higher period elements, does not form $s-p$ hybrids readily [64]. It employs the more loosely bound p-electrons for bonding and the lone pair has mainly s-character. This leads to a pyramidal geometry around phosphorus and prevents efficient interaction of the lone pair with the $\pi$-system. The smaller interaction of the lone pair with the $\pi$-system results in a reduced upward shift of the LUMO and a reduced bandgap compared to PPy. In agreement with this, the IP and EA of PPhos are predicted to be comparable to those of PSi. This is in sharp contrast to the second period of the Periodic Table where the bandgap is largest and IP and EA are smallest for PPy. PPh could therefore be both $\mathrm{p}$ - and n-dopable. We conclude that if well-ordered planar PPh can be made, it will have properties quite different from PPy.

We did not evaluate bandwidths for PPh since it is difficult to identify the $\pi$-orbitals due to the lack of planarity. The phosphorus atoms are slightly above and below the plane defined by the carbon backbone. The C-P-H angle of the central rings is $98.0^{\circ}$. Due to the lack of aromaticity, the intraring $\mathrm{C}=\mathrm{C}$ bonds are short, almost identical to those in PCp and PSi. The intraring single bond is $0.017 \AA$ longer than that in the aromatic PPy. The interring single bond is slightly shorter than in PTh and PPy. This and the low bandgap indicate the presence of conjugation along the chain in $\mathrm{PPh}$. The deviation from planarity in the experimental study is probably due to the bulky phenyl groups at $\mathrm{P}$ and the methyl groups at the carbons.

\subsection{Polythiophene}

Polythiophene has been studied extensively [4 $7,9,11,36,55,65-67]$. Its electronic properties are well established. The bandgap is $2.0 \mathrm{eV}$, it is $\mathrm{n}$ - and p-dopable although the stability of the n-doped form is poor. (A bandgap of 1.7
Table 6

Comparison between theoretical and experimental geometries of quaterphosphole

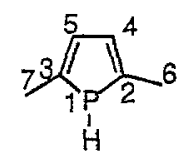

\begin{tabular}{llllll}
\hline & $\mathrm{C}-\mathrm{X}$ & $\mathrm{C}=\mathrm{C}$ & $\mathrm{C}-\mathrm{C}$ & $\Delta$ & $\mathrm{C}-\mathrm{C}^{\prime}$ \\
\hline Theor. & $1.845 / 1.845$ & $1.387 / 1.386$ & 1.449 & 0.062 & 1.453 \\
Exp. & $1.822 / 1.790$ & $1.374 / 1.383$ & 1.445 & 0.062 & 1.43 \\
\hline
\end{tabular}

eV has been observed in alkyl-substituted PTh [68].) Neutral $\mathrm{PTh}$ is an insulator. The highest conductivity reported for the p-doped form of PTh is $2000 \mathrm{~S} / \mathrm{cm} \mathrm{[4].} \mathrm{Our} \mathrm{calculated}$ bandgap is $2.30 \mathrm{eV}$. After correction for the lowering in the condensed phase the calculated value is $0.1 \mathrm{eV}$ from experiment. The IP of PTh is $0.73 \mathrm{eV}$ larger than that of PPy. This explains the better stability of the neutral form of PTh. The EA is $1.59 \mathrm{eV}$ larger than that of PPy. This is in agreement with the finding that PTh can be n-doped while PPy cannot. The HOMO band is slightly wider $(0.08 \mathrm{eV})$ and the conduction band is significantly wider than that of PPy. Hence, the small difference in HOMO bandwidths cannot be the reason for the differences in conductivities of PTh and PPy ( 2000 versus $500 \mathrm{~S} / \mathrm{cm}$ ). Note that the valence band of PTh is actually smaller than that of PPy.

\subsection{Polyselenophene}

The bandgap of polyselenophene was determined electrochemically to be $2.0 \mathrm{eV}$ [15]. This is identical to the bandgap of PTh. The conductivity increased by $10^{5}$ upon doping. The highest conductivity achieved was $3.7 \times 10^{-2} \mathrm{~S} / \mathrm{cm}$. The conductivity in the undoped state was $2.1 \times 10^{-7} \mathrm{~S} / \mathrm{cm}$. The electrical conductivity declines with temperature [15]. It was suggested that the difference in conductivity between PTh and PSe may arise from structural defect related traps as a result of the shorter conjugated chains.

Oligoselenophenes up to hexamers were synthesized and absorption spectra were determined [69]. Selenophene oligomers absorb at wavelengths about $0.3-0.2 \mathrm{eV}$ longer than the corresponding thiophene oligomers. Therefore, one would expect a slightly smaller bandgap for PSe than for PTh. Our calculations predict a difference of $0.15 \mathrm{eV}$. The IP of PSe is calculated to be $0.12 \mathrm{eV}$ lower than that of PTh, the EA is almost the same as that of PTh. HOMO and conduction bandwidths are also the same for PSe as for PTh. There is no reason to expect PSe to be an inferior conductor than PTh, unless the increase in size of the heteroatom leads to larger interchain distances in the solid and prevents hopping between chains. More experimental investigations will most likely eventually increase the conductivity of PSe. 


\subsection{Polytellurophene}

Polytellurophene was obtained by electropolymerization of bitellurophene [16]. Bitellurophene is a yellow-colored solid and relatively stable. The yellow color was attributed to interring conjugation in the planar molecule. The oxidation peak for tellurophene is $0.26 \mathrm{~V}$ lower than that of thiophene and selenophene. PTe was obtained from electropolymerization of bitellurophene as a black film with a conductivity of up to $10^{-6} \mathrm{~S} / \mathrm{cm}$ in the doped form and an intrinsic conductivity of $10^{-12} \mathrm{~S} / \mathrm{cm}$.

Our theoretical results predict a bandgap $0.34 \mathrm{eV}$ smaller for PTe than for PTh. The value of $1.87 \mathrm{eV}$ is, however, still too large for intrinsic conductivity. The IP of PTe is predicted to be $0.24 \mathrm{eV}$ smaller than that of PTh. This in excellent agreement with the 0.26 lower oxidation potential. The EA of PTe is expected to be 0.08 larger than that of PTh. Therefore PTe, like PTh, should be stable in the p-doped form, but not very stable in the n-doped form. The HOMO bandwidth is slightly smaller and the conduction bandwidth is slightly larger than that of PTh. Thus, the lower conductivity measured for PTe compared to PTh is not due to intrinsic electronic properties of PTe. According to our calculations, PTe could be a conductor as good as or better than PTh. Either the material has not been optimized to the same degree as PTh or the size of the heteroatom has an influence on the conductivity in higher period systems.

\subsection{Trends and comparisons between the different polymers}

Table 1 reveals that bandgaps correlate roughly with $\pi$ donor strengths of the bridging groups. With bridging groups from the second period of the Periodic Table, the bandgaps increase in the order PCp $<$ PPy but decrease from PPy to $\mathrm{PFu}$. This demonstrates that $\pi$-donor strength, which shows the same trend, dominates over electronegativity. (PBo is not included in this comparison since its electronic structure is qualitatively different from the other polymers.) Interestingly, in the third period of the Periodic Table the bandgap does not peak for PPh. The bandgap of PPh is almost as small as that for PSi. This can be explained by the fact that phosphole is not aromatic like pyrrole. The phosphorus lone pair is high in s-character and the p-orbital is involved in the bond to hydrogen. As a result the hydrogen is almost perpendicular to the ring plane and the interaction between the lone pair and the $\pi$-system is small.

Comparison of PFu, PTh, PSe and PTe shows that bandgaps decrease in going down the group. The difference is largest between the second and third periods, $0.37 \mathrm{eV}$. The change between the fourth and fifth periods, $0.19 \mathrm{eV}$, is slightly larger than between the third and fourth periods, 0.15 $\mathrm{eV}$. This is in agreement with chemical wisdom that second period elements are the exception in the Periodic Table, due to their ability to effectively hybridize s and p orbitals [64].
It is also well established that third and fourth period elements are more alike than fourth and fifth period elements.

Sexiborole which has two electrons less per ring than the other polymers is quinoid. Although the bond length alternation is largest in PBo, PBo has the smallest bandgap. PPy has the smallest bond alternation and the largest gap. This shows that the bond length alternation, which was shown to strongly influence the bandgap of polythiophene by Andre et al. [66], is of lesser importance when different systems are compared. The $\pi$-donor strength is clearly the dominant effect for the series of systems studied here. Similar conclusions were reached with HF calculations [55].

As discussed elsewhere [33], gas phase IPs and EAs for molecules are underestimated by about $1 \mathrm{eV}$ with our approach. Table 1 shows that solid state IPs of polymers are overestimated by $0.5-0.8 \mathrm{eV}$. There is thus a difference of $1.5-1.8 \mathrm{eV}$ between molecular gas phase and solid state polymer data to be accounted for. This can be rationalized by the solid state polarization energy which has been determined to be about $1.7 \mathrm{eV}$ for conjugated organic molecules [70]. Since the systems studied were fairly large and since no size dependence of the solid state polarization energy was observed, it can be assumed that there is a similar effect for conjugated organic polymers. Thus, the overestimation of polymer IPs in contrast to molecular gas phase IPs gives further support to the existence of a solid state polarization energy for polymers. It also shows that our IPs are flawed by two problems. The first is the systematic error in the IPs for the oligomers, and the second is the neglect of the solid state polarization energy. Thus, we can use these numbers only as a rough guide to determine trends. With this in mind it is encouraging to see that the calculated differences in IPS between PA and PTh $(-0.48 \mathrm{eV})$ and between PA and PPy $(0.25 \mathrm{eV})$ agree reasonably well with the experimental differences of $-0.5 \pm 0.1$ and $0.5 \pm 1 \mathrm{eV}$, respectively.

Comparing IPs and EAs for the different polymers shows that changing the bridging group has a stronger effect on the LUMO (conduction band) than on the HOMO (valence band) energies. The HOMO energies vary only by up to 0.89 $\mathrm{eV}$ while the LUMO energies differ by up to $2.14 \mathrm{eV}$ (excluding $\mathrm{PBo}$ which is not isoelectronic with the other polymers). This might be related to the fact that the HOMO of cyclopentadiene derivatives has a node at the position of the heteroatom. The extrapolated valence bandwidth of $5.96 \mathrm{eV}$ for PA is in good agreement with $6.5 \mathrm{eV}$ obtained with VEH solid state calculations by Brédas [11]. For PTh we obtain a valence bandwidth of $2.75 \mathrm{eV}$. This can be compared to 2.6 $\mathrm{eV}$ [11] with the VEH method, $2.4 \mathrm{eV}$ [5] at LSDA, and $3.07 \mathrm{eV}$ [9] at the MP2 level of theory. The discrepancies are somewhat larger for PPy for which we obtain a valence bandwidth of $3.08 \mathrm{eV}$, while larger values result with VEH, $3.8 \mathrm{eV}$ [11], and at MP2, $3.95 \mathrm{eV}$ [9]. Conduction bandwidths in this work for PTh and PPy are about $1 \mathrm{eV}$ larger than at the MP2 level [9] and the conduction band of PTh is $1.5 \mathrm{eV}$ larger than obtained with the LSD approximation [5]. 
Valence bandwidths of heteroaromatic polymers vary between 1.9 and $4.45 \mathrm{eV}$ and are thus considerably smaller than that of PA. This is due to the avoided crossing between the two highest occupied $\pi$-bands and the splitting of HOMO $-2 \pi$-levels from the valence band. In PA all the occupied $\pi$-levels form one band. PCp has the largest and PTe the smallest valence band of the heterocyclic polymers.

In the absence of avoided crossings, bandwidths reflect the degree of interaction between orbitals along the polymer chain. Larger overlap allows for the stronger delocalization. This is reflected in wider bands in band structure diagrams. In contrast to valence bandwidths, the HOMO bandwidths are very similar for all the polymers investigated here. Therefore, any differences in conductivities of the former systems studied here are most likely not caused by differences in the degree of delocalization.

Although the investigated polymers are very similar in their electronic structures, no systematic relationship between bandwidths and bond alternation or interring distance has been found. The bandwidths seem to be more influenced by the $\pi$-donor strengths than by overlap which turned out to be very similar for all our systems. In the series PPy, PCp, PSi, for instance, the latter two have small bandgaps due to the small effect of the methyl and silyl groups on the HOMO and LUMO energies. They also have the largest bandwidths. For PPy the situation is reversed, the bandgap is large and the bandwidth is small. This can be explained by the fact that $\pi$ donation affects the frontier orbitals more strongly than the lower and higher lying energy levels. Therefore, the same effect that increases bandgaps by pushing HOMO and LUMO apart automatically decreases bandwidths.

The conduction band and the next higher unoccupied band seem to be separate for some of the polymers, e.g. PPy and $\mathrm{PFu}$ (compare Fig. 3) but no appreciable gap seems to develop for others, e.g. PTh, PSe and PTe as shown in Figs. 4 and 5 . There might thus be larger differences in electronic properties of these polymers with respect to n-doping as compared to p-doping.

\section{Conclusions}

Comparison of various heterocyclic organic polymers shows that band structures of all systems with $\pi$-donors are qualitatively very similar. Bandgaps increase in the following order: $\mathrm{PBo}<\mathrm{PSi}<\mathrm{PPh}<\mathrm{PA} \cdot \mathrm{PCp}<\mathrm{PTe}<\mathrm{PSe}<\mathrm{PTh}<$ $\mathrm{PFu}<\mathrm{PPy}$. This roughly reflects the trend in $\pi$-donor strengths. Thus, PPy and PTh, the systems which are most common in the search for conducting polymers because of their chemical stability, are about the worst possible choice as far as bandgaps are concerned.

Weak $\pi$-donor strengths of the heteroatoms leads to small bandgaps. This can be achieved in going to higher periods or to the left in the Periodic Table. PBo stands out because it has two electrons per repeat unit less than the other heteroaromatic polymers. This results in a low lying conduction band and the absence of an avoided crossing in the valence band. $\mathrm{PBo}$ might be a synthetic metal or a semiconductor with an extremely small bandgap, $0.12 \mathrm{eV}$ or less. Small gaps are also predicted for PCp, PSi and PPh. The low bandgap of PPh as compared to PPy can be explained by the pyramidal character of phosphorus and the resulting small overlap of the lone pair with the $\pi$-system. PCp, PSi and PPh have, however, bandgaps well above $1 \mathrm{eV}$ and would have to be doped to become conductive.

Conjugation along the chain, as indicated by similar HOMO bandwidths, is comparable in all systems studied. If the materials could be obtained with similar order and chain lengths, conductivities upon doping could, in principle, be comparable. Like bandgaps, bandwidths seem to be mainly influenced by $\pi$-donor strengths of the heteroatoms. No correlation was found between bandwidths and bond alternation or interring bond distance.

IPs increase in the following order: $\mathrm{PCp}<\mathrm{PPy}<$ $\mathrm{PBo}<\mathrm{PSi}<\mathrm{PPh}<\mathrm{PFu}<\mathrm{PTe}<\mathrm{PSe}<\mathrm{PTh}$. Since $\mathrm{PTh}$ with the highest IP is known to be p-dopable all the other systems should be as well. Since PPy is unstable in the neutral form, the even lower IP of PCp indicates that the neutral form might be unstable.

EAs increase in the order: $\mathrm{PPy}<\mathrm{PFu}<\mathrm{PCp}<\mathrm{PTh}<$ $\mathrm{PSe}<\mathrm{PTe} \ll \mathrm{PSi}<\mathrm{PPh}<\mathrm{PBo}$. Since $\mathrm{PTh}$ is not stable in the $n$-doped state, the systems left of PTh will certainly not be stable in the n-doped form either. The differences in EAs in Group VI are small. Thus, the only reasonable candidates for $\mathrm{n}$-doping are PSi, $\mathrm{PPh}$ and PBo. PBo might be stable in the n-doped form because it will become aromatic and isoelectronic with the other polymers.

The large differences in conductivities upon doping observed experimentally between PTh, PPy, PFu, PSe and PTe are not due to differences in bandgaps and bandwidths, IPs and EAs. They can almost certainly be attributed to different conjugation lengths, disorder and crystal packing effects. For PSe and PTe, we expect that better conductivities will be achieved as the materials become optimized.

\section{Acknowledgements}

The authors thank the Natural Sciences and Engineering Research Council of Canada and Memorial University for financial support. We also thank the Computing and Communications Department of the Memorial University and Digital for computing resources.

\section{References}

[1] G.B. Street, in T.A. Skotheim (ed.), Handbook of Conducting Polymers, Vol. 1, Marcel Dekker, New York, 1986.

[2] K.K. Kanazawa, A.F. Diaz, R.H. Geiss, W.D. Gill, J.F. Kwak, J.A. Logan, J.F. Rabolt, G.B. Street, J. Chem. Soc., Chem. Commun. (1979) 854-855.

[3] Y.E. Whang, S. Miyata, in W.R. Salaneck, I. Lundström, B. Rånby 
(eds.), Conjugated Polymers and Related Materials, Oxford University Press, Oxford, 1993.

[4] J. Roncali, Chem. Rev. 97 (1997) 173-205.

[5] G. Brocks, J. Chem. Phys. 100 (1996) 17 327-17 333.

[6] J.L. Brédas, R. Silbey, D.S. Boudreaux, R.R. Chance, J. Am. Chem. Soc. 105 (1983) 6555-6559.

[7] A.K. Bakhshi, J. Ladik, M. Seel, Phys. Rev. B 35 (1987) 704-712.

[8] J.L. Brédas, J.C. Scott, K. Yakushi, G.B. Street, Phys. Rev. B 30 (1984) 1023-1025.

[9] H.O. Villar, P. Otto, M. Dupuis, J. Ladik, Synth. Met. 59 (1993) $97-$ 110.

[10] G.Zotti, S. Martina, G. Wegner, A.-D. Schlüter, Adv. Mater. 4 (1992) 798-801.

[11] J.L. Brédas, in T.A. Skotheim (ed.), Handbook of Conducting Polymers, Marcel Dekker, New York, 1986, pp. 859-913.

[12] S. Glenis, M. Benz, E. LeGoff, J.L. Schindler, C.R. Kannewurf, M.G. Kanatzidis, J. Am. Chem. Soc. 115 (1993) 12 519-12 525.

[13] M.J. González-Tejera, I. Carrillo, I. Hernández-Fuentes, Synth. Met. 73 (1995) $135-140$.

[14] V. Hernandéz, J.T. López Navarrete, J.L. Marcos, Synth. Met. $41-43$ (1991) 789-792.

[15] S. Glenis, D.S. Ginley, A.J. Frank, J. Appl. Phys. 62 (1987) 190194.

[16] T. Otsubo, S. Inoue, H. Nozoe, T. Jigami, F. Ogura, Synth. Met. 6971 (1995) 537-538.

[17] M.J. Frisch, G.W. Trucks, H.B. Schlegel, P.M.W. Gill, B.G. Johnson, M.A. Robb, J.R. Cheeseman, T. Keith, G.A. Petersson, J.A. Montgomery, K. Raghavachari, M.A. Al-Laham, V.G. Zakrzewski, J.V. Ortiz, J.B. Foresman, C.Y. Peng, P.Y. Ayala, W. Chen, M.W. Wong, J.L. Andres, E.S. Replogle, R. Gomperts, R.L. Martin, D.J. Fox, J.S Binkley, D.J. Defrees, J. Baker, J.P. Stewart, M. Head-Gordon, C. Gonzalez, J.A. Pople, Gaussian 94, Gaussian, Pittsburgh, PA, 1995.

[18] M.J. Frisch, E. Frisch, J.B. Foresman, Gaussian 94 User's Reference, Gaussian, Pittsburgh, PA, 1994-1995.

[19] W. Stevens, H. Basch, J. Krauss, J. Chem. Phys. 81 (1984) 6026.

[20] T.H. Dunning, P.J. Hay, in I. Schaefer (ed.), Modern Theoretical Chemistry, Plenum, New York, 1976, pp. 1-28.

[21] P.J. Hay, W.R. Wadt, J. Chern, Phys. 82 (1985) 270.

[22] W.R. Wadt, P.J. Hay, J. Chem. Phys. 82 (1985) 284.

[23] P.J. Hay, W.R. Wadt, J. Chem. Phys. 82 (1985) 299.

[24] C. Taliani, L.M. Blinov, Adv. Mater. 8 (1996) 353-359.

[25] M. Levy, Phys. Rev. A 52 (1995) 50-52.

[26] R.W. Godby, M. Schliuter, L.J. Sham, Phys. Rev. B 37 (1988) $10159-$ 10175 .

[27] J.P. Perdew, M. Levy, Phys. Rev. Lett. 51 (1983) 1884.

[28] R.K. Nesbet, J. Phys. Chem. 100 (1996) 6104-6106.

[29] L. Fritsche, Physica B 172 (1991) 7-17.

[30] A.R. Williams, U. von Barth, in S. Lundqvist, N.H. March (eds.), Theory of the Inhomogeneous Electron Gas, Plenum, London, 1983.

[31] E.J. Baerends, O.V. Gritsenko, J. Phys. Chem. A 101 (1997) 53835403.

[32] U. Salzner, J.B. Lagowski, P.G. Pickup, R.A. Poirier, J. Phys. Chem., accepted for publication.

[33] U. Salzner, J.B. Lagowski, P.G. Pickup, R.A. Poirier, J. Comput. Chem. 18 (1997) 1943-1953.

[34] R. Hoffmann, Solids and Surfaces: A Chemist's View of Bonding in Extended Structures, VCH, Weinheim, 1988.

[35] G. Horowitz, B. Bachet, A. Yassar, P. Lang, F. Demanze, J.-L. Fave, F. Garnier, Chem. Mater. 7 (1995) 1337-1341.

[36] G. Brocks, P.J. Kelly, R. Car, Synth. Met. 55-57 (1993) 4243-4248.

[37] J.L. Brédas, R.R. Chance, R. Silbey, G. Nicolas, P. Durand, J. Chem. Phys. 75 (1981) 255

[38] A.J. Epstein, in T.A. Skotheim (ed.), Handbook of Conducting Polymers, Vol. 2, Marcel Dekker, New York, 1986.
[39] J.C.R. Fincher, D.L. Peebles, A.J. Heeger, M.A. Druy, Y. Matsamura, A.G. MacDiarmid, H. Shirakawa, S. Ikeda, Solid State Commun. 27 (1978) 489-494.

[40] W.K. Ford, C.B. Duke, A. Paton, J. Chem. Phys. 77 (1982) 45644572.

[41] R.B. Kaner, S.J. Porter, D.P. Nairns, A.G. MacDiarmid, J. Chem. Phys. 90 (1989) 5102-5107.

[42] M.P. Keane, A. Naves de Brito, N. Correira, S. Svensson, L. Karlsson, B. Wannberg, U. Gelius, S. Lunell, W.R. Salaneck, M. Lögdlund, D.B. Swanson, A.G. MacDiarmid, Phys. Rev. B 45 (1992) 63906399.

[43] Y.-S. Lee, M. Kertesz, J. Chem. Phys. 88 (1988) 2609-2617.

[44] L. Rodriguez-Monge, S. Larsson, J. Phys. Chem. 100 (1996) 6298$6303 \mathrm{mmmm}$.

[45] T. Tani, P.M. Grant, W.D. Gill, G.B. Street, T.C. Clarke, Solid State Commun. 33 (1980) 499-503.

[46] A.J. Heeger, in W.R. Salaneck, I. Lundström, B. Rånby (eds.), Conjugated Polymers and Related Materials, Oxford University Press, Oxford, 1993, pp. 28-62.

[47] J.J. Eisch, J. Organomet. Chem. 500 (1995) 101-115.

[48] M. Unverzagt, H.J. Winkler, M. Brock, M. Hoffmann, P.v.R. Schleyer, W. Massa, A. Berndt, Angew. Chem., Int. Ed. Engl. 36 (1997) 853-855.

[49] M. McCann, E.M. Coda, J. Mol. Catal. A 109 (1996) 99-111.

[50] S.Y. Hong, D.S. Marynick, Macromolecules 28 (1995) 4991-4995.

[51] S.Y. Hong, S.J. Kwon, S.C. Kim, J. Chem. Phys. 103 (1995) $1871-$ 1877.

[52] S.Y. Hong, S.J. Kwon, S.C. Kim, D.S. Marynick, Synth. Met. 69-71 (1995) 701-702.

[53] H. Kuroso, M. Kikuchi, I. Ando, J. Polym. Sci. 33 (1995) 769.

[54] G.B. Street, T.C. Clarke, M. Krounbi, K. Kanazawa, V.Lee, P.Pfluger, J.C. Scott, G. Weiser, Mol. Cryst. Liq. Cryst. 83 (1982) 253-264.

[55] P. Otto, Int. J. Quantum Chem. 52 (1994) 353-364.

[56] V. Hernández, J.T. López Navarrete, G. Zotti, M. Veronelli, G. Zerbi, Synth. Met. 69-71 (1995) 391-392.

[57] K. Kaneto, S. Ura, K. Yoshino, Y. Inuishi, Jpn. J. Appl. Phys. 23 (1984) L189, L663.

[58] J. Shinar, S. Ijadi-Maghsoodi, Q.-X. Ni, Y. Pang, T.J. Barton, Synth. Met. 27-29 (1989) C593-C598.

[59] X. Wei, Y. Pang, S. Ijadi-Maghsoodi, T.J. Barton, S. Grigoras, Synth. Met. 41-43 (1991) 1583-1586.

[60] K.S. Wong, S.G. Han, Z.V. Vardeny, J. Shinar, Y. Pang, S. YjadiMaghsoodi, T.J. Barton, S. Grigoras, B. Parbhoo, Appl. Phys. Lett. 58 (1991) 1695-1697.

[61] S. Grigoras, G.C. Lie, T.J. Barton, S. Ijadi-Maghsoodi, Y. Pang, J. Shinar, Z.W. Vardeny, K.S. Wong, S.G. Han, Synth. Met. 49-50 (1992) 293-304.

[62] G. Frapper, M. Kertesz, Synth Met. 55-57 (1993) 4255-4259.

[63] E. Deschamps, L. Ricard, F. Mathey, Angew. Chem., Int. Ed. Engl. 33 (1994) 1158-1161.

[64] W. Kutzelnigg, Angew. Chem., Int. Ed. Engl. 23 (1984) 272.

[65] G.B. Kiliç, L. Toppare, E. Yurtsever, Synth. Met. 78 (1996) 19-25.

[66] J.-A. André, J. Delhalle, J.L. Brédas, Quantum Chemistry Aided Design of Organic Polymers. An Introduction to the Quantum Chemistry of Polymers and Its Applications, World Scientific, London, 1991.

[67] J.L. Brédas, R.L. Elsenbaumer, R.R. Chance, R. Silbey, J. Chem. Phys. 78 (1983) 5656-5662.

[68] T.-A. Chen, R.-D. Rieke, Synth. Met. 60 (1993) 175.

[69] S. Inoue, H. Nakanishi, K. Takimiya, Y. Aso, T. Otsubo, Synth. Met. 84-86 (1997) 341-342.

[70] N. Sato, K. Seki, H. Inokuchi, J. Chem. Soc., Faraday I 77 (1981) $1621-1633$

[71] W.R. Salaneck, H.R. Thomas, C.B. Duke, A. Paton, E.W. Plummer, A.J. Heeger, A.G. MacDiarmid, J. Chem. Phys. 71 (1979) 2044. 